\title{
Linkage Disequilibrium (LD)
}

National Cancer Institute ( $\mathrm{NCl})$

\section{Source}

National Cancer Institute (NCI). Linkage disequilibrium.

Where alleles (DNA markers) occur together more often than can be accounted for by chance because of their physical proximity on a chromosome. Also called LD. 